\title{
Desigualdad social y tendencias de mortalidad por diabetes
}

\author{
Oswaldo Medina-Gómezi,2 e Ismael Seth Medina-Reyes ${ }^{3}$
}

${ }^{1}$ Escuela Nacional de Enfermería y Obstetricia, Universidad Nacional Autónoma de México; ${ }^{2}$ Servicio de Epidemiología, Unidad de Medicina Familiar 15, Instituto Mexicano del Seguro Social (IMSS); ${ }^{3}$ Coordinación de Vigilancia Epidemiológica y Apoyo en Contingencias, IMSS. Ciudad de México, México

\section{Resumen}

Objetivo: Identificar la tendencia de la mortalidad nacional por diabetes según el nivel de marginalidad estatal y municipal. Método: Se realizó un estudio descriptivo con los registros de defunciones por diabetes en mayores de 20 años entre los años 1990 y 2013. Se calculó la tasa de mortalidad nacional estandarizada por edad según la población mundial del año 2000 y para el ámbito estatal se consideró la población nacional proyectada al año 2013. Se realizó un análisis joinpoint para análisis de tendencia. Resultados: Para la población general, el porcentaje de cambio anual entre 1990 y 1996 fue de 2.2, de 1996 a 2005 fue de 4.3 y de 2005 a 2013 fue de 0.1. El mayor incremento entre las mujeres ocurrió entre 1998 y 2005 , mientras que entre los hombres ocurrió entre 1995 a 2006. En el ámbito estatal se encontró un mayor porcentaje de cambio anual entre las localidades con mayor grado de marginación. Conclusiones: La mortalidad por diabetes en las mujeres muestra una disminución importante desde el año 2004; entre los hombres, la mortalidad continúa con una tendencia al alza, lo cual concuerda con la tendencia que la enfermedad ha tenido en los últimos años.

PALABRAS CLAVE: Desigualdades en salud. Marginación social. Diabetes. Mortalidad.

\begin{abstract}
Objective: To identify the trend of national diabetes mortality by level of marginality at the state and municipal levels. Method: $A$ descriptive study was conducted with records of deaths from diabetes in over 20 years from 1990 to 2013. The national mortality rate was calculated standardized by age according to the 2000 world population and the state level 2013 saw the projected naational population by join point analysis for trend analysis was performed. Results: For the general population, the annual percentage change between 1990 and 1996 was 2.2, from 1996 to 2005 was 4.3, and from 2005 to 2013 was 0.1 . The largest increase among women occurred between 1998 and 2005 while among men occurred between 1995 and 2006 . At the state level was found higher annual percentage change between the towns with the highest degree of marginalization. Conclusions: The mortality of diabetes in women shows a significant decrease since 2004, among men, the mortality with a continuous upward trend, consistent with the trend that the disease has had in recent years.
\end{abstract}

KEY WORDS: Health inequity. Social marginalization. Diabetes. Mortality.

\author{
Correspondencia: \\ Oswaldo Medina-Gómez \\ Ermita Iztapalapa, 411 \\ Col. Coyoacán, Del. Prado Churubusco \\ C.P. 04320, Ciudad de México, México \\ E-mail: epired@gmail.com
}

Fecha de recepción: 01-10-2016

Fecha de aceptación: 03-11-2016

DOI://dx.doi.org/10.24875/GMM.17002900
Gac Med Mex. 2017;153:787-793

Contents available at PubMed www.gacetamedicademexico.com 


\section{Introducción}

La diabetes mellitus tipo 2 (DM2) es una enfermedad crónica cuya prevalencia continúa en ascenso en todo el mundo. Se estima que la prevalencia mundial estimada de diabetes en 2000 fue del $2.8 \%$ y se espera que para el año 2030 sea del 4.4\%, lo que representa cerca de 165 millones de personas que serán diagnosticadas con la enfermedad' 1 .

En 2010, la región con mayor prevalencia de DM2 fue Norteamérica, seguida de Europa del Este y el sur de Asia. México ocupó en el mismo año el décimo lugar en el mundo respecto al número de adultos con diabetes, y se espera que ascienda al séptimo lugar ${ }^{2}$.

El cambio demográfico debido al aumento de la proporción de personas mayores de 65 años ha contribuido al incremento de la prevalencia de diabetes en todo el mundo ${ }^{3}$.

Si bien se ha planteado la existencia de la regulación genética entre el metabolismo de lípidos, la acción y la resistencia de la insulina, así como el incremento de marcadores inflamatorios, como la proteína $C$ reactiva y la interleucina 6 , que contribuyen biológicamente en la predisposición de los individuos a desarrollar diabetes en algún momento de su vida- ${ }^{4-7}$, también son sus determinantes sociales los que establecen las diferencias en la incidencia, la prevalencia y la mortalidad de la enfermedad en los distintos grupos sociales ${ }^{8-11}$. En todo el mundo se ha identificado que las condiciones socioeconómicas, como la posición social, la educación y los ingresos, son determinantes importantes que contribuyen al incremento en la mortalidad relacionada con la diabetes ${ }^{11-14}$.

El impacto de la enfermedad se expresa de manera diferenciada entre los grupos sociales. Existe evidencia de que las tasas de incidencia han ido en aumento principalmente entre los que viven en comunidades de bajos ingresos, y sobre todo entre las mujeres pobres ${ }^{15-17}$.

En México, la DM2 es la primera causa de mortalidad y muestra un incremento progresivo en los últimos años ${ }^{18}$, siendo la primera causa de muerte, de años perdidos por muerte prematura, de años vividos con discapacidad y de años de vida saludable perdidos $^{19}$.

Diversos esfuerzos se han realizado en México en materia de prevención y promoción de la salud ${ }^{20-22}$, y es por ello que el objetivo del presente estudio fue analizar los cambios en las tendencias de mortalidad por DM2 en México según el nivel de marginalidad durante el periodo 1990-2013, utilizando el modelo de regresión joinpoint.

\section{Método}

Se realizó un estudio descriptivo a partir de fuentes secundarias de información correspondientes al periodo 1990-2013 para las 31 entidades federativas y el Distrito Federal. Se estudiaron todas las defunciones registradas en el Sistema de Información en Salud de la Secretaría de Salud de México durante el periodo de tiempo analizado, con registro de causa básica de defunción codificada con los códigos E11-E14 de la Décima Revisión de la Clasificación Internacional de Enfermedades (CIE-10) en personas mayores de 20 años, y las defunciones reportadas por la Dirección General de Información en Salud.

La tasa de mortalidad cruda por DM2 se calculó como el número de defunciones que ocurren dividido entre el número de personas en un año determinado según el registro realizado por el Consejo Nacional de Población (CONAPO), y se realizó un ajuste de tasas al ámbito nacional por método directo por edad utilizando la población mundial del año 2010 reportada por la Organización Mundial de la Salud. Para los Estados, se consideró la población de referencia reportada para el país para 2013.

Se utilizó el paquete estadístico Joinpoint Regression Program 4.2.0.2 para el análisis de tendencias de las tasas ajustadas de mortalidad por diabetes en los ámbitos nacional y estatal para identificar y describir la ocurrencia de cambios en un periodo de tiempo a través de la tendencia de los datos. Para la construcción del modelo se eligió la transformación logarítmica. El programa selecciona el modelo final utilizando la prueba de permutación y criterio de información bayesiano reportando el número mínimo y máximo de joinpoints, señalando el modelo más parsimonioso.

La tasa de cambio para cada tendencia se prueba para determinar si el cambio es significativamente distinto de cero. Cada tendencia en el modelo final se describe por un porcentaje de cambio anual (PCA) con un intervalo de confianza del 95\% (IC 95\%). Si el IC 95\% no contiene el cero, el PCA se considera estadísticamente significativo.

Finalmente, se realizó el análisis de joinpoint por sexo y por el grado de marginalidad municipal de residencia registrado en el certificado de defunción, reportados por el Consejo Nacional de Evaluación de la Política de Desarrollo Social (CONEVAL) para el año 2012. 
Tabla 1. Tasa de mortalidad por diabetes mellitus tipo 2 ajustada por edad según Estado de residencia, México, 1990-2013

\begin{tabular}{|c|c|c|c|c|c|c|}
\hline \multirow[t]{2}{*}{ Estado } & \multicolumn{2}{|c|}{1990} & \multicolumn{2}{|c|}{2000} & \multicolumn{2}{|c|}{2013} \\
\hline & TM & IC 95\% & TM & IC $95 \%$ & TM & IC $95 \%$ \\
\hline Aguascalientes & 82.5 & $72.02-94.4$ & 95.3 & 86.1-105.2 & 114.2 & $106.2-122.6$ \\
\hline Baja California & 123.3 & $113.2-134.2$ & 120.6 & $113.5-128.0$ & 108.9 & 103.9-114.0 \\
\hline Baja California Sur & 94.5 & $77.2-114.4$ & 100.9 & $86.4-117.2$ & 95.6 & 85.2-106.9 \\
\hline Campeche & 44.1 & $36.1-53.4$ & 61.2 & $53.0-70.4$ & 110.7 & $101.7-120.3$ \\
\hline Coahuila & 133.3 & $124.5-142.7$ & 130.6 & $123.7-137.8$ & 131.5 & 126.1-136.9 \\
\hline Colima & 63.3 & $52.9-75.4$ & 80.9 & $70.7-92.2$ & 126.3 & $116.0-137.4$ \\
\hline Chiapas & 27.5 & $24.7-30.5$ & 63.2 & $59.4-67.1$ & 100.9 & $97.0-104.9$ \\
\hline Chihuahua & 134.9 & $126.8-143.4$ & 98.0 & $92.7-103.6$ & 104.4 & 100.1-108.7 \\
\hline Distrito Federal & 142.1 & $142.1-146.7$ & 127.8 & $124.7-131.1$ & 105.6 & $103.2-108$ \\
\hline Durango & 95.1 & $86.6-104.3$ & 89.8 & $83-97.2$ & 103.4 & $97.5-109.7$ \\
\hline Guanajuato & 70.0 & $66.1-74.2$ & 106.3 & $102.2-110.5$ & 137.9 & $134-141.9$ \\
\hline Guerrero & 26.6 & $24-29.5$ & 53.8 & $50.3-57.5$ & 108.7 & $104.4-113.2$ \\
\hline Hidalgo & 46.7 & $42.5-51.1$ & 72.1 & $67.4-77$ & 102.3 & $97.6-107$ \\
\hline Jalisco & 77.1 & 73.6-80.7 & 97.0 & $93.7-100.5$ & 111.6 & 108.6-114.6 \\
\hline México & 32.7 & $31.5-34$ & 121.7 & $118.7-124.9$ & 134.8 & $132.4-135.5$ \\
\hline Michoacán & 49.6 & $46.5-53$ & 84.6 & 80.9-88.5 & 126.2 & $126.2-130.2$ \\
\hline Morelos & 60.7 & $54.7-67.3$ & 86.5 & 80.4-93.1 & 122.3 & 116.3-128.4 \\
\hline Nayarit & 58.6 & $51.5-66.4$ & 71.4 & $64.4-79$ & 74.2 & $68.3-80.5$ \\
\hline Nuevo León & 95.7 & $90-101.9$ & 89.3 & 84.9-93.8 & 99.8 & $96.3-103.4$ \\
\hline Oaxaca & 29.0 & $28.7-26.2$ & 58.1 & $55.2-52$ & 117.1 & $100.8-97$ \\
\hline Puebla & 61.3 & $57.9-64.8$ & 101.1 & $97.2-105$ & 144.6 & $140.7-148.5$ \\
\hline Querétaro & 62.5 & $55.2-70.5$ & 92.9 & $85.5-100.7$ & 107.0 & $100.7-113.5$ \\
\hline Quintana Roo & 41.2 & $30.6-54.3$ & 57.4 & $48-68.3$ & 120.7 & $111.3-130.9$ \\
\hline San Luis Potosí & 51.9 & $47.5-56.5$ & 70.8 & $66.2-75.6$ & 99.6 & $95.1-104.4$ \\
\hline Sinaloa & 68.6 & $63-74.6$ & 80.4 & $75.4-85.7$ & 81.8 & $77.8-86$ \\
\hline Sonora & 100.5 & $92.2-109.6$ & 97.8 & $91.7-104.3$ & 98.3 & $93.6-103.1$ \\
\hline Tabasco & 41.0 & $36.1-46.3$ & 87.9 & 81.6-94.6 & 154.0 & $147.2-161$ \\
\hline Tamaulipas & 101.8 & $95.2-108.7$ & 108.9 & $103.4-114.8$ & 120.2 & $115.6-124.9$ \\
\hline Tlaxcala & 66.6 & $58.4-75.7$ & 106.1 & $97.2-115.7$ & 158.7 & 149.6-168.2 \\
\hline Veracruz & 54.5 & $51.9-57.1$ & 82.6 & $79.8-85.5$ & 130.4 & $127.4-133.4$ \\
\hline Yucatán & 61.1 & $55-67.9$ & 65.6 & $60.5-71$ & 89.9 & 84.9-95 \\
\hline Zacatecas & 38.8 & $34-44.2$ & 67.6 & 61.9-73.6 & 97.9 & $92-104.1$ \\
\hline
\end{tabular}

IC 95\%: intervalo de confianza del 95\%; TMA: tasa de mortalidad por 100,000 habitantes ajustada por edad.

\section{Resultados}

Durante el periodo analizado se reportaron $1,279,765$ defunciones a causa de DM2 en personas de 20 años y más. El $46.2 \%(\mathrm{~N}=591,105)$ correspondieron al sexo masculino y el $53.8 \%(\mathrm{~N}=688,660)$ al sexo femenino; 2016 certificados no especificaron el sexo o la edad de la persona, y por tal motivo se eliminaron del estudio. 


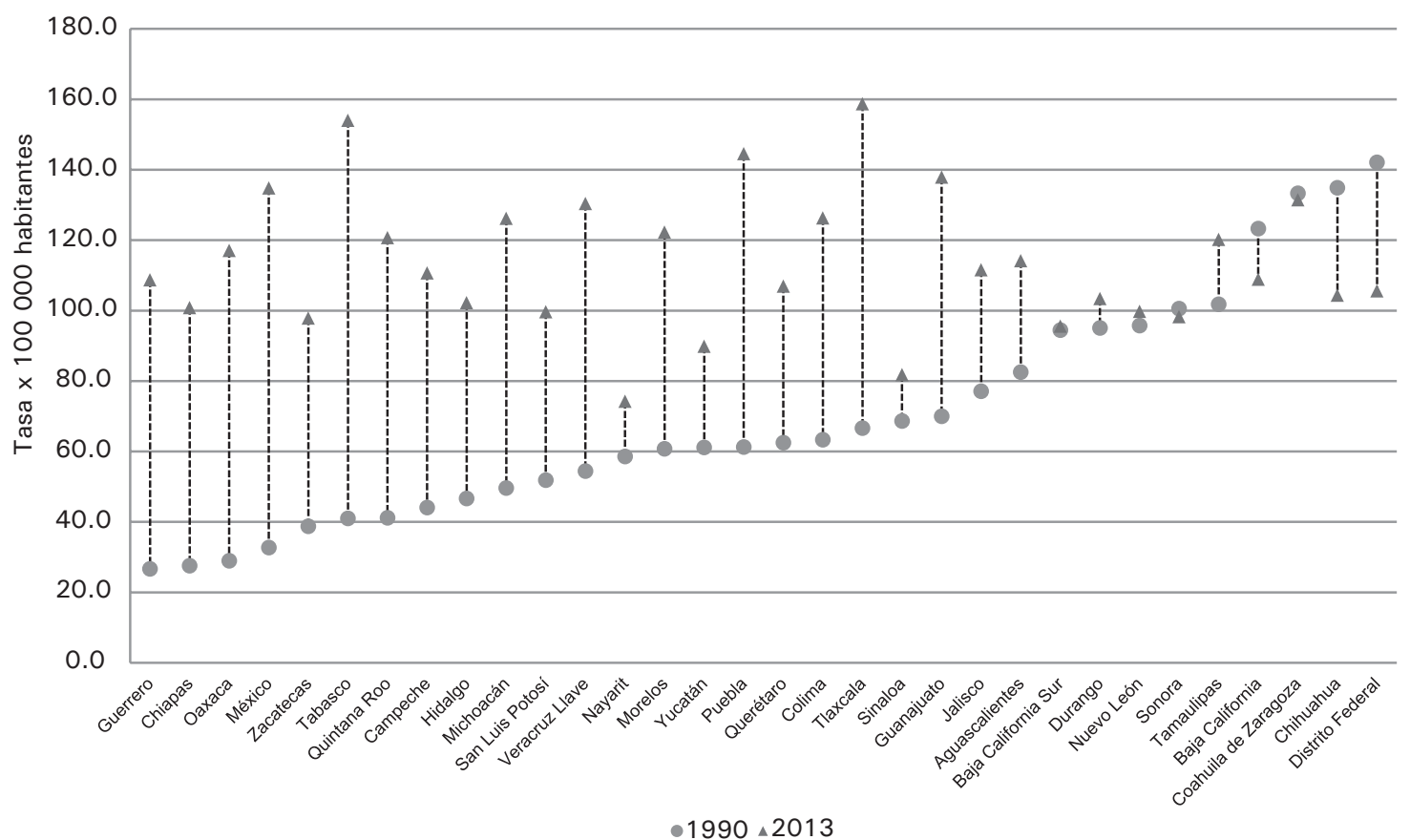

Figura 1. Tasa de mortalidad ajustada por entidad federativa, México, 1990 y 2013.

Tabla 2. Porcentaje de cambio anual de la mortalidad por diabetes mellitus tipo 2 en la población de 20 años y más, por sexo y nivel de marginalidad del municipio de residencia, México 1990-2013

\begin{tabular}{|c|c|c|c|c|c|}
\hline Cohorte & Segmentos reportados & Periodo & PCA & IC 95\% & p \\
\hline \multirow[t]{3}{*}{ Nacional } & 1 & 1990-1996 & 2.2 & $1.1-3.4$ & $<0.001$ \\
\hline & 2 & 1996-2005 & 4.3 & $3.5-5.0$ & $<0.001$ \\
\hline & 3 & $2005-2013$ & 0.1 & $-0.6-0.8$ & 0.75 \\
\hline \multirow[t]{3}{*}{ Hombres } & 1 & 1990-1995 & 1.3 & $-1.0-3.6$ & 0.24 \\
\hline & 2 & 1995-2006 & 4.5 & $4.0-5.1$ & $<0.001$ \\
\hline & 3 & $2006-2013$ & 0.9 & $0.1-1.7$ & 0.02 \\
\hline \multirow[t]{3}{*}{ Mujeres } & 1 & 1990-1998 & 2.2 & $1.2-3.1$ & 0.001 \\
\hline & 2 & $1998-2005$ & 4.4 & $3.2-5.6$ & 0.001 \\
\hline & 3 & $2005-2013$ & -0.4 & $-1.0-0.3$ & 0.25 \\
\hline \multicolumn{6}{|c|}{ Nivel de marginalidad del municipio de residencia } \\
\hline Muy bajo & 1 & 1990-1994 & 2.7 & $0.3-5.2$ & 0.03 \\
\hline Bajo & 2 & 1994-2005 & 6.9 & $6.3-7.5$ & $<0.001$ \\
\hline Medio & 3 & 2005-2013 & 2.3 & $1.5-3.1$ & $<0.001$ \\
\hline Alto & 1 & 1990-2005 & 7.1 & $6.8-7.5$ & $<0.001$ \\
\hline \multirow[t]{6}{*}{ Muy alto } & 2 & 2005-2013 & 4.3 & $3.5-5.2$ & $<0.001$ \\
\hline & 1 & 1990-2004 & 8.1 & $7.7-8.4$ & $<0.001$ \\
\hline & 2 & 2004-2013 & 5.0 & $4.3-5.7$ & $<0.001$ \\
\hline & 1 & 1990-2009 & 8.5 & $8.2-8.8$ & $<0.001$ \\
\hline & 2 & $2009-2013$ & 4.8 & $1.7-7.9$ & 0.004 \\
\hline & 1 & 1990-2013 & 10.6 & $10.2-10.9$ & $<0.001$ \\
\hline
\end{tabular}

IC 95\%: intervalo de confianza del 95\%; PCA: porcentaje de cambio anual.

Las tasas estatales de mortalidad por DM2 ajustadas por edad se describen en la tabla 1. Las diferencias de las tasas de mortalidad de 1990 a 2013 reflejan un incremento heterogéneo de las tasas de mortalidad por DM2, sobresaliendo Tabasco con mayor diferencia (Fig. 1).
En el modelo joinpoint para la tendencia de mortalidad nacional por diabetes se identificaron tres puntos de cambio en el tiempo. El primer periodo corresponde a 1990-1996, el cual presenta un PCA de 2.2 estadísticamente significativo; el siguiente cambio se identificó en 1996-2005, con un PCA de 4.3 (IC 95\%: 3.5-5.0; 
Tabla 3. Porcentaje de cambio anual de la mortalidad por diabetes mellitus tipo 2 por entidad federativa, México, 1990-2013

\begin{tabular}{|c|c|c|c|c|c|c|c|c|c|}
\hline & Período & PCA & IC 95\% & $p$ & & Período & PCA & IC 95\% & $p$ \\
\hline Aguascalientes & $1990-2013$ & 1.5 & $0.8-2.1$ & $<0.001$ & Nayarit & $1990-2010$ & 3.1 & $2.6-3.7$ & $<0.001$ \\
\hline \multirow[t]{3}{*}{ Baja California } & 1990-1993 & -4.6 & $-11.6-3.1$ & 0.22 & & $2010-2013$ & -8.1 & $-17.2-2$ & 0.11 \\
\hline & 1993-2006 & 1 & $0.1-1.9$ & 0.04 & Nuevo León & 1990-1994 & -4.7 & $-10.7-1.7$ & 0.14 \\
\hline & $2006-2013$ & -2 & $-4-0$ & 0.53 & & 1994-2005 & 3.1 & $1.4-4.7$ & $<0.001$ \\
\hline Baja California Sur & $1990-2013$ & 0.8 & $0.3-1.3$ & 0.03 & & $2005-2013$ & -1.3 & $-3.5-0.9$ & 0.24 \\
\hline Campeche & $1990-2013$ & 3.9 & $3.4-4.5$ & $<0.001$ & Oaxaca & $1990-2004$ & 7.3 & $6.7-7.8$ & $<0.001$ \\
\hline \multirow[t]{3}{*}{ Coahuila } & 1990-1996 & -1.6 & $-3.5-0.4$ & 0.11 & & $2004-2013$ & 3.2 & $2.2-4.2$ & $<0.001$ \\
\hline & 1996-2005 & 3.3 & $2-4.6$ & $<0.001$ & Puebla & 1990-1995 & 1.7 & $-1.1-4.5$ & 0.23 \\
\hline & $2005-2013$ & -2.5 & $-3.8--1.3$ & $<0.001$ & & $1995-2003$ & 7.7 & $5.9-9.5$ & $<0.001$ \\
\hline Colima & $1990-2013$ & 2.9 & $2.5-3.3$ & $<0.001$ & & 2003-2013 & 1.1 & $0.1-2.1$ & 0.03 \\
\hline \multirow[t]{2}{*}{ Chiapas } & 1990-2002 & 8.5 & $7.7-9.2$ & $<0.001$ & Querétaro & 1990-1996 & 1.2 & $-0.9-3.4$ & 0.23 \\
\hline & $2002-2013$ & 3.4 & $2.6-4.2$ & $<0.001$ & & 1996-1999 & 10.2 & $-2.8-24.9$ & 0.12 \\
\hline \multirow[t]{3}{*}{ Chihuahua } & 1990-1992 & -14.6 & $-27.7-0.8$ & 0.06 & & 1999-2008 & 2.4 & $1-3.8$ & 0.002 \\
\hline & 1992-2008 & 1.5 & $0.8-2.3$ & $<0.001$ & & $2008-2013$ & -1.3 & $-4-1.6$ & 0.30 \\
\hline & $2008-2013$ & -3.9 & $-7.4--0.2$ & 0.04 & Quintana Roo & $1990-2013$ & 4.5 & $3.8-5.2$ & $<0.001$ \\
\hline \multirow[t]{3}{*}{ Distrito Federal } & 1990-1994 & -4.1 & $-7.5--0.6$ & 0.02 & San Luis Potosí & 1990-1997 & 2.1 & $0.4-3.8$ & 0.02 \\
\hline & 1994-2010 & 1.1 & $0.6-1.6$ & $<0.001$ & & 1997-2005 & 5.8 & $4-7.5$ & $<0.001$ \\
\hline & $2010-2013$ & -8.1 & $-13.2--2.8$ & 0.006 & & $2005-2013$ & 0.4 & $-1-1.7$ & 0.58 \\
\hline \multirow[t]{3}{*}{ Durango } & $1990-2001$ & 0.6 & $-0.8-2$ & 0.40 & Sinaloa & 1990-1995 & 0.2 & $-2.5-3$ & 0.88 \\
\hline & $2001-2006$ & 6.7 & $0-13.8$ & 0.05 & & $1995-2003$ & 4.4 & $2.7-6.1$ & $<0.001$ \\
\hline & $2006-2013$ & -4.1 & $-6.7--1.5$ & 0.005 & & 2003-2013 & -1.3 & $-2.3--0.4$ & 0.01 \\
\hline \multirow[t]{2}{*}{ Guanajuato } & 1990-2005 & 4.2 & $3.6-4.7$ & $<0.001$ & Sonora & $1990-2013$ & 0.1 & $-0.2-0.5$ & 0.37 \\
\hline & $2005-2013$ & 0.6 & $-0.7-2$ & 0.35 & Tabasco & $1990-2004$ & 7 & $5.9-8.1$ & $<0.001$ \\
\hline \multirow[t]{2}{*}{ Guerrero } & 1990-1996 & 9 & $6.4-11.6$ & $<0.001$ & & $2004-2013$ & 2.8 & $0.8-4.8$ & 0.009 \\
\hline & $1996-2013$ & 5.7 & $5.1-6.2$ & $<0.001$ & Tamaulipas & 1990-1994 & -2.4 & $-5.1-0.3$ & 0.07 \\
\hline \multirow[t]{2}{*}{ Hidalgo } & $1990-2007$ & 4.4 & $4-4.8$ & $<0.001$ & & 1994-2003 & 3.2 & $2.2-4.2$ & $<0.001$ \\
\hline & $2007-2013$ & 1 & $-0.7-2.7$ & 0.25 & & 2003-2013 & -0.0004 & $-0.7-0.6$ & 0.91 \\
\hline \multirow[t]{2}{*}{ Jalisco } & 1990-2005 & 2.7 & $2.2-3.1$ & $<0.001$ & Tlaxcala & $1990-2013$ & 3.9 & $3.6-4.3$ & $<0.001$ \\
\hline & $2005-2013$ & -0.4 & $-1.5-0.8$ & 0.50 & Veracruz & 1990-1998 & 3.6 & $2.6-4.7$ & $<0.001$ \\
\hline \multirow[t]{2}{*}{ México } & 1990-1992 & 61.2 & $34.8-92.6$ & $<0.001$ & & 1998-2002 & 7.9 & $3.1-12.9$ & 0.003 \\
\hline & $1992-2013$ & 1.8 & $1.4-2.3$ & $<0.001$ & & $2002-2013$ & 2.8 & $2.1-3.4$ & $<0.001$ \\
\hline \multirow[t]{2}{*}{ Michoacán } & 1990-2005 & 5.3 & $4.9-5.6$ & $<0.001$ & Yucatán & $1990-2000$ & 0.8 & $-0.3-2$ & 0.15 \\
\hline & $2005-2013$ & 2 & $1.1-3$ & $<0.001$ & & $2000-2003$ & 10.8 & $-4.3-28.4$ & 0.15 \\
\hline \multirow[t]{2}{*}{ Morelos } & 1990-2008 & 4.1 & $3.6-4.5$ & $<0.001$ & & 2003-2013 & $<0.001$ & $-1.1-1.2$ & 0.96 \\
\hline & $2008-2013$ & 0.3 & $-2.9-3.6$ & 0.83 & Zacatecas & $1990-2013$ & 3.5 & $2.7-4.2$ & $<0.001$ \\
\hline
\end{tabular}


$\mathrm{p}<0.01$ ); y el tercer periodo corresponde a 2005-2013, con un PCA de 0.1 (IC 95\%: -0.6-0.8) (Tabla 2).

En las mujeres, en 1990-1998 el PCA fue de 2.2 (IC 95\%: 1.2-3.1); en 1998-2005 fue de 4.4 (IC 95\%: $3.2-5.6) ; \quad y$ en 2005-2013 fue de -0.4 , no estadísticamente significativo $(p=0.25)$.

En los hombres, en 1990-1995 el PCA fue de 1.3 ( $p=0.24$ ); en 1995-2006 fue de 4.5 (IC 95\%: 4.0-5.1); $y$ en 2006-2013 fue de 0.9 (IC 95\%: 0.1-1.7).

El análisis de joinpoint según el nivel de marginalidad del municipio de residencia reportado en el certificado de defunción muestra que el PCA se incrementa en todos los niveles; sin embargo, es mayor en los municipios con muy alta marginación (PCA $=10.6$; IC 95\%: 10.2-10.9) y abarca dicho incremento todo el periodo de tiempo de estudio (Tabla 2).

La tasa de mortalidad por DM2 en la entidad con el mayor índice de marginalidad (Guerrero) es 1.03 veces mayor que en la entidad de menor nivel de marginalidad (Distrito Federal), mientras que el Estado con el mayor porcentaje de población con ingresos inferiores a la línea de bienestar (Chiapas) presenta una tasa de mortalidad 1.01 veces mayor que aquella en mejores condiciones (Nuevo León).

La entidad federativa con el menor porcentaje de acceso a servicios de salud (Puebla) presenta una tasa de mortalidad por DM2 1.3 veces mayor respecto al de mayor acceso (Campeche).

Al considerar el análisis de joinpoint por cada Estado sobresale el incremento de la mortalidad por DM2 en el Estado de México entre los años 1990 y 1992, siendo el mayor nivel estatal durante los años analizados (Tabla 3).

\section{Discusión}

La mortalidad por diabetes en México afecta a ambos sexos, lo cual difiere con lo encontrado en otros países $^{23,24}$. Entre las mujeres, desde el año 2004 la mortalidad por diabetes muestra una disminución importante; por otro lado, entre los hombres la mortalidad continúa con una tendencia al alza, lo cual concuerda con la tendencia que la enfermedad ha tenido en los últimos años ${ }^{25}$.

En el caso de la diabetes, se ha demostrado que las personas que llevan una dieta adecuada logran un mejor control metabólico. Sin embargo, el control de factores de riesgo cardiovascular en los pacientes diabéticos es precario en México ${ }^{26}$, lo cual incide directamente en un incremento en la mortalidad de la población.
Es necesario considerar la limitación de utilizar las defunciones reportadas, pues estas suelen ser susceptibles de una mala calidad del registro principalmente porque en México existe un sobrerregistro de diabetes en los certificados de defunción ${ }^{27,28}$.

Sin embargo, al analizar la mortalidad por DM2 con el método joinpoint se pueden estudiar los cambios en las tendencias en los ámbitos nacional y estatal, permitiendo conocer si las modificaciones son significativas, además de identificar la heterogeneidad de los cambios en las tendencias de la mortalidad.

Los resultados obtenidos abren nuevas interrogantes acerca de la efectividad de las políticas de salud centradas en el individuo, y se hace necesario realizar estudios posteriores que analicen la distribución y los determinantes sociales, como el acceso a servicios de salud y la marginalidad, entre otros, que contribuyen a los patrones de cambio en la mortalidad por DM2, mismos que resultan ser grandes obstáculos para la detección, la atención y la prevención de esta enfermedad ${ }^{29-31}$.

El análisis de las tendencias de mortalidad por el método joinpoint resulta ser muy útil para monitorear los cambios de una enfermedad a través del tiempo, lo cual puede contribuir al diseño de nuevas estrategias en salud permitiendo identificar las variaciones en las tendencias de mortalidad de la enfermedad y reconocer si dichos cambios son significativos.

\section{Bibliografía}

1. Zimmet $P$, Alberti K, Shaw J. Global and societal implications of the diabetes epidemic. Nature. 2001;414:782-7.

2. Shaw JE, Sicree RA, Zimmet PZ. Global estimates of the prevalence of diabetes for 2010 and 2030. Diabetes Res Clin Pract. 2010;87:4-14.

3. Wild S, Roglic G, Green A, et al. Global prevalence of diabetes: estimates for the year 2000 and projections for 2030. Diabetes Care. 2004;27:1047-53.

4. Emanuelli B, Glondu M, Filloux C, et al. The potential role of SOCS-3 in the interleukin-10-induced desensitization of insulin signaling in pancreatic beta-cells. Diabetes. 2004;53:S97-S103.

5. Muller-Wieland D, Knebel B, Avci A, et al. Insulin-regulated transcription factors: molecular link between insulin resistance and cardiovascular risk factors. Int J Obes Metab Disord. 2001:25(Suppl 1):35-7.

6. Pradhan AD, Manson JE, Rifai N, et al. C-reactive protein, interleukin 6, and risk of developing type 2 diabetes mellitus. JAMA. 2001;286:327-34.

7. Banerjee M, Saxena M. Interleukin-1 (IL-1) family of cytokines: role in type 2 diabetes. Clin Chim Acta. 2012;413:1163-70.

8. Hsu CC, Lee CH, Wahlqvist ML, et al. Poverty increases type 2 diabetes incidence and inequality of care despite universal health coverage. Diabetes Care. 2012;35:2286-92.

9. Marmot M, Allen JJ. Social determinants of health equity. Am J Public Heatlh. 2014;104(Suppl 4):S517-9.

10. Blas E, Kurup AS. Equity, social determinants and public health programmes.Geneve: World Health Organization; 2010.

11. Espelt A, Arriola L, Borrell C, et al. Socioeconomic position and type 2 diabetes mellitus in Europe 1999-2009: a panorama of inequalities. Curr Diabetes Rev. 2011;7:148-58.

12. Dray-Spira R, Gary-Webb TL, Brancati FL. Educational disparities in mortality among adults with diabetes in the U.S. Diabetes Care. 2010; 33:1200-5.

13. Espelt $A$, Kunst $A E$, Palència $L$, et al. Twenty years of socio-economic inequalities in type 2 diabetes mellitus prevalence in Spain, 1987-2006. Eur J Public Health. 2012;22:765-71. 
14. Borrell C, Azlor E, Rodríguez-Sanz M, et al. Trends in socioeconomic mortality inequalities in a southern European urban setting at the turn of the $21^{\text {st }}$ century. J Epidemiol Community Health. 2008;62:258-66.

15. Lee TC, Glynn RJ, Peña JM, et al. Socioeconomic status and incident type 2 diabetes mellitus: data from the Women's Health Study. PLoS One. 2011;6:e27670.

16. Agardh $E$, Allebeck $P$, Hallqvist $J$, et al. Type 2 diabetes incidence and socio-economic position: a systematic review and meta-analysis. Int $J$ Epidemiol. 2011;40:804-8

17. Raphael D, Anstice S, Raine K, et al. The social determinants of the incidence and management of type 2 diabetes mellitus: are we prepared to rethink our questions and redirect our research activities? Leadership in Health Services. 2003;16:10-20.

18. Rull JA, Aguilar-Salinas CA, Rojas R, et al. Epidemiology of type 2 diabetes in Mexico. Arch Med Res. 2005;36:188-96.

19. Rodríguez-Abrego G, Escobedo J, Zurita B, et al. Muerte prematura y discapacidad en los derechohabientes del Instituto Mexicano del Seguro Social. Salud Pública Méx. 2007;49:132-43.

20. León-Mazón MA, Arauj-Mendoza GJ, Linos-Vázquez ZZ. Eficacia del programa de educación en diabetes (DiabetIMSS) en los parámetros clínicos y bioquímicos. Rev Med Inst Mex Seguro Soc. 2012;51:74-9.

21. Gutiérrez G, Pérez-Cuevas R, Levy S, et al. Strengthening preventive care programs: a permanent challenge for healthcare systems; lessons from PREVENIMSS México. Public Health. 2010;10:417.

22. Instituto Mexicano del Seguro Social. Guía técnica para otorgar atención médica en el módulo DiabetIMSS a derechohabientes con diagnósticos de diabetes mellitus, en Unidades de Medicina Familiar. México: IMSS; 2009.
23. Sandín M, Espelt A, Escolar-Pujolar A, et al. Desigualdades de género y diabetes mellitus tipo 2: la importancia de la diferencia. Av Diabetol. 2011;27:78-87.

24. Dávila-Cervantes CA, Agudelo-Botero M, Gloria-Hernández LE. Diabetes en México y Colombia: análisis de la tendencia de años de vida perdidos. Rev Salud Pública. 2011;13:560-71.

25. Davila C, Pardo A. Diabetes mellitus: aporte al cambio en esperanza de vida en México 1990, 2000 y 2010. Rev Salud Pública. 2014;16:910-23.

26. Escobedo de la Peña J, Reinoso J, Flores L, et al. Encuesta nacional del tratamiento y control metabólico y de los factores de riesgo cardiovascular de los pacientes con diabetes mellitus tipo 2, atendidos por especialistas en medicina interna. Med Int Mex. 2010;26:449-56.

27. Estadísticas de mortalidad en México: muertes registradas en el año 2001. Salud Pública Méx. 2002;44:565-81.

28. Hernández B, Ramírez-Villalobos D, Romero M, et al. Assessing quality of medical death certification: concordance between gold standard diagnosis and underlying cause of death in selected Mexican hospitals. Popul Health Metr. 2011;9:38.

29. Regidor E, Guallar-Castillón P, Gutiérrez-Fisac JL, et al. Socioeconomic variation in the magnitude of the association between self-rated health and mortality. Ann Epidemiol. 2010;20:395-400.

30. Saydah S, Lochner K. Socioeconomic status and risk of diabetes-related mortality in the U.S. Public Health Rep. 2010;125:377-88.

31. Jaffiol C, Thomas F, Bean K, et al. Impact of socioeconomic status on diabetes and cardiovascular risk factors: results of a large French survey. Diabetes Metab. 2013;39:56-62. 